\title{
Stress Affection of Two Scale Truncated Generalized Logistic Parameters with Progressive Censoring
}

\author{
Salma Omar Bleed \\ Head of Department of Statistics, College of Science \\ El-Asmariya University, Zliten-Libya \\ SalmaBleed@yahoo.com
}

Abdallah Mohamed Abd Elfattah

Agamy Higher Institute for Administrative Sciences, Ministry of Higher Education

Professor of Mathematical Statistics Institute of Statistical Studies \& Research, Cairo University

a_afattah@staff.cu.edu.eg

\begin{abstract}
This paper deals with non-Bayesian estimation problem of constant-stress Accelerated Life Tests (ALTs) when the lifetime of the items follow truncated Generalized Logistic Distribution (GLD). Some considerations on inference based on the use of asymptotically normality of the ML estimators are presented considering the stress effects on the two scale parameters of the truncated GLD with a k-level constant-stress ALT under progressive type-I censored grouped data. The EM algorithm method is used to obtain the estimators of the unknown parameters. In addition, estimator of the two scale parameters, reliability function under usual conditions and Fisher information matrix of the estimators are given. Finally, we present a Simulation Study to illustrate the proposed procedure.
\end{abstract}

Keywords and phrases: Truncated Generalized Logistic Distribution; Constant-stress Accelerated Life Test; EM algorithm method; Fisher Information Matrix; Progressive Type-I Censored Grouped Data.

\section{Introduction}

With today's highly reliable components, we are often unable to obtain a reasonable amount of test data under normal use condition. For this reason, ALT is the reasonable procedure to be applied. ALT is used to determine the reliability of a product in a short period of time by accelerating the use environment. Nelson (1990) has indicated that the stress can be applied in various ways, commonly used methods are step stress and constant stress ALT. In real life, many products such as, insulations, bearing, electronic components, semiconductors, and microelectronics run at a constant stress (i.e., run under constant temperature, voltage, load, and etc). CSALT is the most comely adopted ALT so that it is the easiest way to run and estimate reliability information for high reliability and long lifetime products under usual conditions. In CSALT, the stress is kept at a constant level of stress throughout the life test, i.e., each unit is run at a constant high stress level until the occurrence of failure or the observation is censored. Since, CSALT is a widely used in assessing the reliability of products. There is abundant literature on how to design CSALT. They differ in the assumed lifetime distribution, censoring scheme, and test condition. For example, Meeker and Nelson (1975) proposed statistically optimal plans for CSALT which involved only two stress levels. Meeker and Hahn (1977) considered optimal allocation of test units to accelerated stress conditions with the objective of minimizing the estimate of the product reliability under usual condition. Nelson (1990) reviewed statistically optimal and compromise plans for the single stress ALT planning 
problem. Another approach adopted by Yang (1994) and Yang and Jin (1994) to overcome the problem of Meeker and Nelson (1975) was to add test constraints involving expected minimum number of failures to be observed at the middle stress levels. Tang, et al. (2002) presented two alternative ways of CSALT planning with three stress levels that optimize the sample and the sample locations. Tang and $\mathrm{Xu}$ (2005) considered a framework with two conflicting objectives in the planning of ALT, meeting a desired level of statistical precision for an estimate of interest, and meeting a cost target for conducting the test. Ding et al. (2010) considered the design of ALT sampling plans under type-I progressive interval censoring with random removals and assumed the lifetime of products follows a Weibull distribution. Attia, et al. (2011a,b) obtained MLE and discussed optimum test plans of the Generalized Logistic (GL) parameters under both type-I and type-II censoring data. It is assumed that the stress affects only the scale GL parameter. The term Generalized Logistic (GL) distribution is used as the name for several different families of probability distributions [see Johnson, et al. (1995)]. The main feature of the GL distribution is that new parameters were introduced to control both location and scale. It allows for a greater degree of flexibility and it is expected to be useful in many more practical situations Nadarajah and Kotz (2005).

Truncated distributions arise when sample selection is not possible in some sub-region of the sample space. The GLD is considered inappropriate for modeling lifetime data because left hand side of its distribution extends to negative infinity, and this could conceivably result in modeling negative times-to-failure. This has necessitated the use of truncated GLD truncated at point zero for modeling lifetime data.

This paper, considers CSALT under truncated GLD using progressive type-I censored grouped data. In addition to this introductory section this paper includes other Sections too. In Section 2, the model and assumptions are described. Section 3 presents the estimation method. EM algorithm method for obtaining the estimators of the unknown parameters are described in Section 4. In Section 5 the method developed has been illustrated using the data simulated from the proposed models.

\section{Model and Assumptions}

The generalized logistic distribution has been addressed by Molenberghs and Verbeke (2011), which had the following cumulative distribution function

$$
F(x)=1-\left(1+\frac{\alpha_{2}}{\theta} e^{\alpha_{1} x}\right)^{-\theta}, \quad-\infty<x<\infty,
$$

where $\theta$ is a positive shape parameter, and $\alpha_{1}, \alpha_{2}$ are positive scale parameters. In this paper, we use the truncated GLD (truncated at point zero for modeling lifetime data), Thus the cumulative distribution function is given by the following form

$$
F\left(x, \alpha_{1}, \alpha_{2}\right)=1-\left(1+\frac{\alpha_{2}}{\theta}\right)^{\theta}\left(1+\frac{\alpha_{2}}{\theta} e^{\alpha_{1 x}}\right)^{-(\theta+1)}, x, \theta, \alpha_{q}>0, q=1,2
$$

We assume the test is done at high stresses $v_{j}, j=1, \ldots, k$ where $v_{1}<v_{2}<\ldots<v_{k}$ and $v_{u}$ be the design stress; that is the stress level under usual conditions, where $v_{u}<$ $v_{j}, j=1,2, \ldots, k$. For the testing procedure, a total of $N$ units are divided into $n_{1}$, $n_{2}, \ldots, n_{k}$ units where $\sum_{j=1}^{k} n_{j}=N$. Each $n_{j}, j=1,2, \ldots, k$ units in the experiment are run at a pre-specified constant stress $v_{j}, j=1,2, \ldots, k$. The life of a test unit at each 
stress is described by the truncated three-parameter GLD. Thus, the cumulative distribution function of the lifetime of a test unit under k-level step-stress test is

$F\left(x_{i j}, \alpha_{1 j}, \alpha_{2 j}\right)=1-\left(1+\frac{\alpha_{2 j}}{\theta}\right)^{\theta}\left(1+\frac{\alpha_{2 j}}{\theta} e^{\alpha_{1 j} x_{i j}}\right)^{-(\theta+1)}, x_{i j}, \theta, \alpha_{q j}>0, q=1,2$

The scale parameters $\alpha_{q j}, j=1,2, \ldots, k, q=1,2$ at the stress level $v_{j}, j=1,2, \ldots, k$ of a test unit is a power law function of stress [see, Mann, Schafer and Singurwalla(1974)], given by

$$
\alpha_{q j}=c_{q} v_{j}^{p}
$$

where $c_{q}, p$ constants to be estimated. Progressively censored sampling is an important method for lifetime studies. This method allows the experimenter to save time and cost and it is useful when the items being tested are very expensive. Let us consider there are $k$-stage constant stress ALT scheme with progressive Type-I interval censoring. In this case, the test will be carrying out as follows: $n$ units are simultaneously placed on a life test at stress level $v_{1}$, and run until the time $x_{1}$. At this time, the number of failed units $r_{1}$ are counted and $R_{1}$ surviving units are removed from the test; starting from the time $x_{1}$, the $\left(n-r_{1}-R_{1}\right)$ non-removed surviving units are put to a stress level $v_{2}, v_{1},<v_{2}$, and run until the time $x_{2}$. At this time, the number of failed units $r_{2}$ are counted and $R_{2}$ surviving units are removed from the test. Starting from the time $x_{2}$, the $\left(n-r_{1}-\right.$ $R_{1}-r_{2}-R_{2}$ ) non-removed surviving units are put to a stress level $v_{3}, v_{2},<v_{3}$ and run until the time $x_{3}$. At this time, the number of failed units $r_{3}$ are counted and $R_{3}$ surviving units are removed from the test, and so on. At the time $x_{k}$, the number of failed units $r_{k}$ are counted and the remaining surviving units $R_{k}=n-\sum_{j=1}^{k} r_{j}-\sum_{j=1}^{k-1} R_{j}$ are all removed, thereby terminating the test, [Balakrishnan and Aggarwala(2000)].

\section{Estimation Method}

Suppose a type-I progressively censored grouped sample is collected as described in Section 2 and by assuming that the experiment is done under $k$-stage constant stress level, the number of failed units $r_{j}, \quad j=1,2, \ldots, k$ which are observed while the testing in the interval $\left(x_{j-1}, x_{j}\right)$ at stress $v_{j}, j=1,2, \ldots, k$ are random variables with the fact that $r_{j} \mid r_{j-1}, \ldots, r_{1} \sim$ Binomial $\left[m_{j}, F_{j}(x)\right]$, where $m_{j}=N-\sum_{i=1}^{j-1} r_{i}-\sum_{i=1}^{j-1} R_{i}$ is the number of non-removal surviving units at the beginning of the $j^{\text {th }}$ stage, and $F_{j}(x)=$ $\frac{F\left(x_{j}\right)-F\left(x_{j-1}\right)}{1-F\left(x_{j-1}\right)}$. Therefore, the cdf of the lifetime distribution at the stress level $v_{j}, j=$ $1,2, \ldots, k$ and in the inspection interval $\left(x_{j-1}, x_{j}\right)$ will take the following form

$F\left(x_{i j}, \alpha_{1 j}, \alpha_{2 j}\right)=1-\left(1+\frac{\alpha_{2 j}}{\theta}\right)^{\theta}\left(1+\frac{\alpha_{2 j}}{\theta} e^{\alpha_{1 j} x_{i j}}\right)^{-(\theta+1)}, x_{i j}, \theta, \alpha_{q j}>0, q=1,2$

where $\alpha_{q j}$ is defined by Eq.(1). Therefore, the likelihood function is then

$$
L=\prod_{j=1}^{k}\left[F\left(x_{j}\right)-F\left(x_{j-1}\right)\right]^{r_{j}} \cdot\left[1-F\left(x_{j}\right)\right]^{R_{j}} .
$$

In the special case where the intervals are of equal length, so monitoring and censoring occurs periodically, say $x_{j=} j \cdot x$ [Aggarwala (2001)]. Therefore, the likelihood function (2) can be re-written as the following form 


$$
L=\prod_{j=1}^{k}[F(j x)-F((j-1) x)]^{r_{j}} \cdot[1-F(j x)]^{R_{j}} .
$$

Substituting for $\alpha_{q j}$ the expression from (1) involving $c_{1}, c_{2}, p$ the likelihood function can be written as

$$
\begin{aligned}
& L=\prod_{j=1}^{k}\left(1+\frac{c_{2} v_{j}^{p}}{\theta}\right)^{\theta R_{j}}\left(1+\frac{c_{2} v_{j}^{p}}{\theta} e^{j c_{1} v_{j}^{p} x}\right)^{-\theta R_{j}}\left[\left(1+\frac{c_{2} v_{j}^{p}}{\theta}\right)^{\theta}\left(1+\frac{c_{2} v_{j}^{p}}{\theta} e^{(j-1) c_{1} v_{j}^{p} x}\right)^{-\theta}-\right. \\
& \left.\left(1+\frac{c_{2} v_{j}^{p}}{\theta}\right)^{\theta}\left(1+\frac{c_{2} v_{j}^{p}}{\theta} e^{j c_{1} v_{j}^{p} x}\right)^{-\theta}\right]^{r_{j}} .
\end{aligned}
$$

Therefore, the log-likelihood function is

$\ln L=\sum_{j=1}^{k}\left\{R_{j} \ln A_{j}(\varnothing)+\left(r_{j}+R_{j}\right) \ln A_{j}(\varepsilon)+r_{j} \ln \left[A_{j-1}(\varnothing)-A_{j}(\varnothing)\right]\right\}$,

where

$\emptyset=\left(c_{1}, c_{2}, p, \theta\right), \underline{\varepsilon}=\left(c_{2}, p, \theta\right), \underline{\omega}=\left(c_{1}, p\right)$,

$A_{j}(\varepsilon)=\left(1+\frac{c_{2} v_{j}^{p}}{\theta}\right)^{\theta} A_{j}(\varnothing)=\left(1+B_{j}(\varnothing)\right)^{-\theta}, B_{j}(\varnothing)=\frac{c_{2} v_{j}^{p}}{\theta} e^{j c_{1} v_{j}^{p} x}$,

$A_{j-1}(\varnothing)=\left(1+B_{j-1}(\varnothing)\right)^{-\theta}, B_{j-1}(\varnothing)=\frac{c_{2} v_{j}^{p}}{\theta} e^{(j-1) c_{1} v_{j}^{p} x}$.

From $\frac{\partial \ln L}{\partial c_{1}}=0, \frac{\partial \ln L}{\partial c_{2}}=0, \frac{\partial \ln L}{\partial p}=0$, and $\frac{\partial \ln L}{\partial \theta}=0$ we find the maximum likelihood estimators $\left(\hat{c}_{1}, \hat{c}_{2}, \hat{p}, \hat{\theta}\right)$ by solving the following four likelihood equations, $\sum_{j=1}^{k} j R_{j} B_{j}(\widehat{\varnothing}) A_{j}^{\frac{1}{\hat{\theta}}}(\widehat{\varnothing})=r_{j}\left(A_{j-1}(\widehat{\varnothing})-A_{j}(\widehat{\varnothing})\right)^{-1}\left[j B_{j}(\widehat{\varnothing}) A_{j}^{\frac{1}{\widehat{\vartheta}^{+1}}}(\widehat{\varnothing})-(j-\right.$

1) $\left.B_{j-1}(\widehat{\varnothing}) A_{j-1}^{\frac{1}{\widehat{\theta}+1}}(\widehat{\varnothing})\right]$

$\sum_{j=1}^{k}\left\{\left(r_{j}+R_{j}\right) v_{j}^{\hat{p}} A_{j}^{\frac{-1}{\hat{\theta}}}(\hat{\varepsilon})-\right.$

$\left.\frac{\widehat{\theta} R_{j}}{\hat{c}_{2}} B_{j}(\widehat{\varnothing}) A_{j}^{\frac{1}{\hat{\theta}}}(\widehat{\varnothing})\right\}=\frac{r_{j}\left[B_{j}(\widehat{\varnothing}) A_{j}^{\frac{1}{\hat{\theta}+1}}(\widehat{\varnothing})-(j-1) B_{j-1}(\widehat{\varnothing}) A_{j-1}^{\frac{1}{\theta}+1}(\widehat{\varnothing})\right]}{\left(A_{j-1}(\widehat{\varnothing})-A_{j}(\widehat{\varnothing})\right)}$

$\sum_{j=1}^{k}\left\{\frac{R_{j} B_{j}(\widehat{\phi}) A_{j}(\widehat{\omega})}{A_{j}^{\frac{-1}{\widehat{\theta}}}(\widehat{\phi})}-\right.$

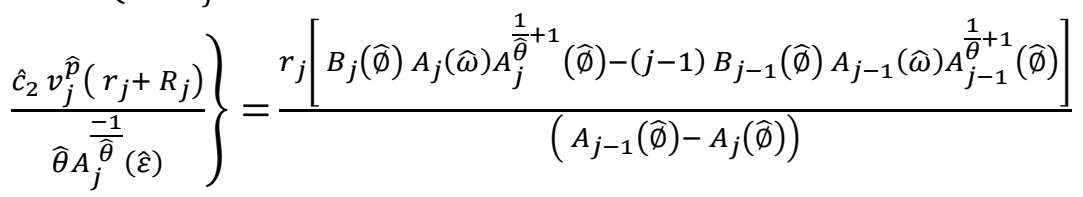




$$
\begin{aligned}
& \sum_{j=1}^{k}\left\{\left(r_{j}+R_{j}\right)\left[\ln \left(1+\frac{\hat{c}_{2} v_{j}^{\widehat{p}}}{\widehat{\theta}}\right)-\frac{\hat{c}_{2} v_{j}^{\widehat{p}}}{\widehat{\widehat{\theta}} A_{j}^{\frac{1}{\hat{\theta}}}(\hat{\varepsilon})}\right]-R_{j}\left[\ln \left(1+B_{j}(\widehat{\varnothing})\right)-\right.\right. \\
& \left.\left.B_{j}(\widehat{\varnothing}) A_{j}^{\frac{1}{\hat{\theta}}}(\widehat{\varnothing})\right]\right\}=\frac{r_{j}\left[\left[A_{j}(\widehat{\phi})\left[\ln \left(1+B_{j}(\widehat{\varnothing})\right)-B_{j}(\widehat{\varnothing}) A_{j}^{\frac{1}{\hat{\theta}}}(\widehat{\phi})\right]-Q_{j-1}(\widehat{\varnothing})\right]\right]}{\left(A_{j-1}(\widehat{\varnothing})-A_{j}(\widehat{\phi})\right)}
\end{aligned}
$$

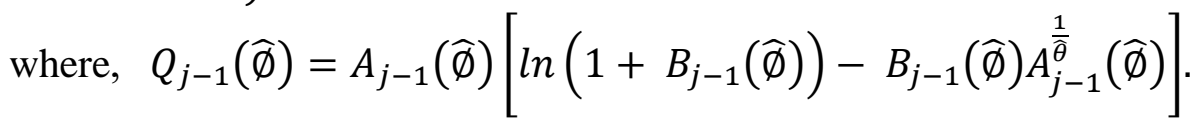

To predict the value of the two scale parameters $\alpha_{1 u}$ and $\alpha_{2 u}$ under the usual condition stress $v_{u}$, the invariance property of MLE is used. Thus, the MLE of $\hat{\alpha}_{1 u}, \hat{\alpha}_{2 u}$ and $\hat{R}_{u}\left(x_{0}\right)$ at the lifetime $x_{0}$ under the usual condition stress $v_{u}$ respectively, are given by the following equations

$$
\hat{\alpha}_{1 u}=\hat{c}_{1} v_{u}^{\hat{p}}, \quad \hat{\alpha}_{2 u}=\hat{c}_{2} v_{u}^{\hat{p}}, \quad \hat{R}_{u}\left(x_{0}\right)=\left(1+\frac{\hat{\alpha}_{2 u}}{\hat{\theta}}\right)\left(1+\frac{\hat{\alpha}_{1 u}}{\hat{\theta}} e^{\hat{\alpha}_{1 u} x_{0}}\right)^{-\widehat{\theta}}
$$

For inference on $\widehat{\Phi}=\left(\hat{c}_{1}, \hat{c}_{2}, \hat{p}, \hat{\theta}\right)$ we usually use the asymptotical normality of the maximum likelihood estimators given by

$$
\widehat{\Phi}=\left(\hat{c}_{1}, \hat{c}_{2}, \hat{p}, \hat{\theta}\right) \sim N\left(\underline{\Phi}, I_{0}^{-1}\right),
$$

Where

and

$$
I_{0}=\left(\begin{array}{llll}
I_{11} & I_{12} & I_{13} & I_{14} \\
I_{21} & I_{22} & I_{23} & I_{24} \\
I_{31} & I_{32} & I_{33} & I_{34} \\
I_{41} & I_{42} & I_{43} & I_{44}
\end{array}\right)
$$

$$
\begin{aligned}
& I_{11}=-\frac{\partial^{2} \ln L(\Phi)}{\partial c_{1}^{2}}=\theta x^{2} \sum_{j=1}^{k} v_{j}^{2 p}\left\{j^{2} R_{j} B_{j}(\varnothing) A_{j}^{\frac{1}{\theta}}(\varnothing)\left[1-B_{j}(\varnothing) A_{j}^{\frac{1}{\theta}}(\varnothing)\right]+r_{j}\left[A_{j-1}(\varnothing)-\right.\right. \\
& \left.A_{j}(\varnothing)\right]^{-1}\left\{\left[j^{2} B_{j}(\varnothing) A_{j}^{1+\frac{1}{\theta}}(\varnothing)\left[(\theta+1) B_{j}(\varnothing) A_{j}^{\frac{1}{\theta}}(\varnothing)-1\right]-(j-1)^{2} B_{j-1}(\varnothing) A_{j-1}^{1+\frac{1}{\theta}}(\varnothing)[(\theta+\right.\right. \\
& \text { 1) } \left.\left.B_{j-1}(\varnothing) A_{j-1}^{\frac{1}{\theta}}(\varnothing)-1\right]\right]+\theta\left[A_{j-1}(\varnothing)-A_{j}(\varnothing)\right]^{-1}\left[j B_{j}(\varnothing) A_{j}^{1+\frac{1}{\theta}}(\varnothing)-(j-\right. \\
& \text { 1) } \left.\left.\left.B_{j-1}(\varnothing) A_{j-1}^{1+\frac{1}{\theta}}(\varnothing)\right]^{2}\right\}\right\} \text {, } \\
& I_{12}=-\frac{\partial^{2} \ln L(\Phi)}{\partial c_{1} \partial c_{2}}=\frac{\theta x}{c_{2}} \sum_{j=1}^{k} v_{j}^{2 p}\left\{j R_{j} B_{j}(\varnothing) A_{j}^{\frac{1}{\theta}}(\varnothing)\left[1-B_{j}(\varnothing) A_{j}^{\frac{1}{\theta}}(\varnothing)\right]+r_{j}\left[A_{j-1}(\varnothing)-\right.\right. \\
& \left.A_{j}(\varnothing)\right]^{-1}\left\{\left[j B_{j}(\varnothing) A_{j}^{1+\frac{1}{\theta}}(\varnothing)\left[(\theta+1) B_{j}(\varnothing) A_{j}^{\frac{1}{\theta}}(\varnothing)-1\right]-(j-1) B_{j-1}(\varnothing) A_{j-1}^{1+\frac{1}{\theta}}(\varnothing)[(\theta+\right.\right.
\end{aligned}
$$




$$
\begin{aligned}
& \text { 1) } \left.\left.B_{j-1}(\varnothing) A_{j-1}^{\frac{1}{\theta}}(\varnothing)-1\right]\right]+\theta\left[A_{j-1}(\varnothing)-A_{j}(\varnothing)\right]^{-1}\left[B_{j}(\varnothing) A_{j}^{1+\frac{1}{\theta}}(\varnothing)-\right. \\
& \left.\left.\left.B_{j-1}(\varnothing) A_{j-1}^{1+\frac{1}{\theta}}(\varnothing)\right]\left[j B_{j}(\varnothing) A_{j}^{1+\frac{1}{\theta}}(\varnothing)-(j-1) B_{j-1}(\varnothing) A_{j-1}^{1+\frac{1}{\theta}}(\varnothing)\right]\right\}\right\}, \\
& I_{13}=-\frac{\partial^{2} \ln L(\Phi)}{\partial c_{1} \partial p}=\theta x \sum_{j=1}^{k} v_{j}^{p} \ln v_{j}\left\{j R_{j} B_{j}(\varnothing) A_{j}(\omega) A_{j}^{\frac{1}{\theta}}(\varnothing)\left[1-B_{j}(\varnothing) A_{j}^{\frac{1}{\theta}}(\varnothing)\right]+\right. \\
& r_{j}\left[A_{j-1}(\varnothing)-A_{j}(\varnothing)\right]^{-1}\left\{\left[j B_{j}(\varnothing) A_{j}(\omega) A_{j}^{1+\frac{1}{\theta}}(\varnothing)\left[(\theta+1) B_{j}(\varnothing) A_{j}^{\frac{1}{\theta}}(\varnothing)-1\right]-(j-\right.\right. \\
& \text { 1) } \left.B_{j-1}(\varnothing) A_{j-1}(\omega) A_{j-1}^{1+\frac{1}{\theta}}(\varnothing)\left[(\theta+1) B_{j-1}(\varnothing) A_{j-1}^{\frac{1}{\theta}}(\varnothing)-1\right]\right]+\theta\left[A_{j-1}(\varnothing)-\right. \\
& \left.A_{j}(\varnothing)\right]^{-1}\left[B_{j}(\varnothing) A_{j}(\omega) A_{j}^{1+\frac{1}{\theta}}(\varnothing)-B_{j-1}(\varnothing) A_{j-1}(\omega) A_{j-1}^{1+\frac{1}{\theta}}(\varnothing)\right]\left[j B_{j}(\varnothing) A_{j}^{1+\frac{1}{\theta}}(\varnothing)-(j-\right. \\
& \text { 1) } \left.\left.\left.B_{j-1}(\varnothing) A_{j-1}^{1+\frac{1}{\theta}}(\varnothing)\right]\right\}\right\} \text {, } \\
& I_{14}=-\frac{\partial^{2} \ln L(\Phi)}{\partial c_{1} \partial \theta}=x \sum_{j=1}^{k} v_{j}^{p}\left\{j R _ { j } B _ { j } ( \varnothing ) A _ { j } ^ { \frac { 1 } { \theta } } ( \varnothing ) \left[B_{j}(\varnothing) A_{j}^{\frac{1}{\theta}}(\varnothing)-\frac{1}{\theta} \ln A_{j}(\varnothing)-\ln (1+\right.\right. \\
& \left.\left.B_{j}(\varnothing)\right)\right]+r_{j}\left[A_{j-1}(\varnothing)-A_{j}(\varnothing)\right]^{-1}\left\{j B_{j}(\varnothing) A_{j}^{1+\frac{1}{\theta}}(\varnothing)[1+(\theta+1)[\ln (1+\right. \\
& \left.\left.\left.B_{j}(\varnothing)\right)-B_{j}(\varnothing) A_{j}^{\frac{1}{\theta}}(\varnothing)\right]+\frac{1}{\theta} \ln A_{j}(\varnothing)\right]+\left[j B_{j}(\varnothing) A_{j}^{1+\frac{1}{\theta}}(\varnothing)-(j-\right. \\
& \text { 1) } \left.B_{j-1}(\varnothing) A_{j-1}^{1+\frac{1}{\theta}}(\varnothing)\right]\left[\theta \left(A_{j}(\varnothing)\left(\ln \left(1+B_{j}(\varnothing)\right)+B_{j}(\varnothing) A_{j}^{\frac{1}{\theta}}(\varnothing)\right)-A_{j-1}(\varnothing)(\ln (1+\right.\right. \\
& \left.\left.\left.\left.\left.\left.B_{j}(\varnothing)\right)+B_{j-1}(\varnothing) A_{j-1}^{\frac{1}{\theta}}(\varnothing)\right)\right)-1\right]\right\}\right\} \text {, } \\
& I_{22}=-\frac{\partial^{2} \ln L(\underline{\phi})}{\partial c_{2}^{2}}=\frac{\theta}{c_{2}^{2}} \sum_{j=1}^{k}\left\{\left(\frac{c_{2}}{\theta}\right)^{2}\left(r_{j}+R_{j}\right) v_{j}^{2 p} A_{j}^{\frac{-2}{\theta}}(\varepsilon)-R_{j} B_{j}^{2}(\varnothing) A_{j}^{\frac{2}{\theta}}(\varnothing)-\right. \\
& r_{j}\left[A_{j-1}(\varnothing)-A_{j}(\varnothing)\right]^{-1}\left\{\left[B_{j}(\varnothing) A_{j}^{1+\frac{1}{\theta}}(\varnothing)\left[1-(\theta+1) B_{j}(\varnothing) A_{j}^{\frac{1}{\theta}}(\varnothing)\right]-\right.\right. \\
& \left.B_{j-1}(\varnothing) A_{j-1}^{1+\frac{1}{\theta}}(\varnothing)\left[1-(\theta+1) B_{j-1}(\varnothing) A_{j-1}^{\frac{1}{\theta}}(\varnothing)\right]\right]+\left[\theta \left[A_{j-1}(\varnothing)-\right.\right. \\
& \left.\left.A_{j}(\varnothing)\right]^{-1}\left(B_{j}(\varnothing) A_{j}^{1+\frac{1}{\theta}}(\varnothing)-B_{j-1}(\varnothing) A_{j-1}^{1+\frac{1}{\theta}}(\varnothing)\right)-1\right]\left[B_{j}(\varnothing) A_{j}^{1+\frac{1}{\theta}}(\varnothing)-\right. \\
& \left.\left.\left.B_{j-1}(\varnothing) A_{j-1}^{1+\frac{1}{\theta}}(\varnothing)\right]\right\}\right\} \text {, }
\end{aligned}
$$




$$
\begin{aligned}
& I_{23}=-\frac{\partial^{2} \ln L(\underline{\Phi})}{\partial c_{2} \partial p}=\sum_{j=1}^{k} \ln v_{j}\left\{\left(r_{j}+R_{j}\right) v_{j}^{p} A_{j}^{\frac{-1}{\theta}}(\varepsilon)\left[\frac{c_{2} v_{j}^{p}}{\theta} A_{j}^{\frac{-1}{\theta}}(\varepsilon)-1\right]+\right. \\
& \frac{\theta}{c_{2}}\left[R_{j} B_{j}(\varnothing) A_{j}(\omega) A_{j}^{\frac{1}{\theta}}(\varnothing)\left(1-B_{j}(\varnothing) A_{j}^{\frac{1}{\theta}}(\varnothing)\right)-r_{j}\left[A_{j-1}(\varnothing)-A_{j}(\emptyset)\right]^{-1}\left\{\left[B_{j}(\varnothing) A_{j}(\omega) A_{j}^{1+\frac{1}{\theta}}(\varnothing)[1-\right.\right.\right. \\
& \left.\left.(\theta+1) B_{j}(\varnothing) A_{j}^{\frac{1}{\theta}}(\varnothing)\right]-B_{j-1}(\varnothing) A_{j-1}^{1+\frac{1}{\theta}}(\varnothing) A_{j-1}(\omega)\left[1-(\theta+1) B_{j-1}(\varnothing) A_{j-1}^{\frac{1}{\theta}}(\varnothing)\right]\right]- \\
& \theta\left[A_{j-1}(\varnothing)-A_{j}(\varnothing)\right]^{-1}\left(B_{j}(\varnothing) A_{j}^{1+\frac{1}{\theta}}(\varnothing)-B_{j-1}(\varnothing) A_{j-1}^{1+\frac{1}{\theta}}(\varnothing)\right)\left(B_{j}(\varnothing) A_{j}^{1+\frac{1}{\theta}}(\varnothing) A_{j}(\omega)-\right. \\
& \left.\left.\left.\left.B_{j-1}(\varnothing) A_{j-1}^{1+\frac{1}{\theta}}(\varnothing) A_{j-1}(\omega)\right)\right\}\right]\right\} \\
& I_{24}=-\frac{\partial^{2} \ln L(\underline{\Phi})}{\partial c_{2} \partial \theta}=\sum_{j=1}^{k}\left\{\frac{1}{\theta^{2}}\left(r_{j}+R_{j}\right) v_{j}^{p} A_{j}^{\frac{-1}{\theta}}(\varepsilon)\left[\ln A_{j}(\varepsilon)+\theta \ln \left(1+\frac{c_{2} v_{j}^{p}}{\theta}\right)-\frac{c_{2} v_{j}^{p}}{A_{j}(\varepsilon)}\right]+\right. \\
& \frac{R_{j} B_{j}(\varnothing) A_{j}^{\frac{1}{\theta}}(\varnothing)}{c_{2}}\left[B_{j}(\varnothing) A_{j}^{\frac{1}{\theta}}(\varnothing)-\frac{1}{\theta} \ln A_{j}(\varnothing)-\ln \left(1+B_{j}(\varnothing)\right)\right]- \\
& \frac{\theta r_{j}}{c_{2}\left(A_{j-1}(\varnothing)-A_{j}(\varnothing)\right)}\left\{\frac { 1 } { \theta ^ { 2 } } \left[B _ { j } ( \varnothing ) A _ { j } ^ { 1 + \frac { 1 } { \theta } } ( \varnothing ) \left[\theta(\theta+1)\left(B_{j}(\varnothing) A_{j}^{\frac{1}{\theta}}(\varnothing)-\ln \left(1+B_{j}(\varnothing)\right)\right)-\right.\right.\right. \\
& \left.\ln A_{j}(\varnothing)-\theta\right]-B_{j-1}(\varnothing) A_{j-1}^{1+\frac{1}{\theta}}(\varnothing)\left[\theta(\theta+1)\left(B_{j-1}(\varnothing) A_{j-1}^{\frac{1}{\theta}}(\varnothing)-\ln \left(1+B_{j-1}(\varnothing)\right)\right)-\right. \\
& \left.\left.\ln A_{j-1}(\varnothing)-\theta\right]\right]-\left(B_{j}(\varnothing) A_{j}^{1+\frac{1}{\theta}}(\varnothing)-B_{j-1}(\varnothing) A_{j-1}^{1+\frac{1}{\theta}}(\varnothing)\right)\left[\left(A_{j-1}(\varnothing)-\right.\right. \\
& \left.A_{j}(\varnothing)\right)^{-1}\left(A_{j}(\varnothing)\left(\ln \left(1+B_{j}(\varnothing)\right)-B_{j}(\varnothing) A_{j}^{\frac{1}{\theta}}(\varnothing)\right)-A_{j-1}(\varnothing)\left(\ln \left(1+B_{j}(\varnothing)\right)-\right.\right. \\
& \left.\left.\left.\left.\left.B_{j-1}(\varnothing) A_{j-1}^{\frac{1}{\theta}}(\varnothing)\right)\right)-\frac{1}{\theta}\right]\right\}\right\} \\
& I_{33}=-\frac{\partial^{2} \ln L(\underline{\Phi})}{\partial p^{2}}=\theta \sum_{j=1}^{k} \ln v_{j}^{2}\left\{R_{j} B_{j}(\varnothing) A_{j}^{\frac{1}{\theta}}(\emptyset)\left[A_{j}^{2}(\omega)\left(1-B_{j}(\varnothing) A_{j}^{\frac{1}{\theta}}(\varnothing)\right)+j c_{1} v_{j}^{p}\right]-\right. \\
& \frac{\left(r_{j}+R_{j}\right) c_{2} v_{j}^{p}}{\theta A_{j}^{\frac{1}{\theta}}(\varepsilon)}\left(1-\frac{c_{2} v_{j}^{p}}{\theta A_{j}^{\frac{1}{\theta}}(\varepsilon)}\right)-r_{j}\left[A_{j-1}(\varnothing)-A_{j}(\varnothing)\right]^{-1}\left\{\left[B _ { j } ( \varnothing ) A _ { j } ^ { 1 + \frac { 1 } { \theta } } ( \varnothing ) \left[A_{j}^{2}(\omega)[1-\right.\right.\right. \\
& \left.\left.(\theta+1) B_{j}(\varnothing) A_{j}^{\frac{1}{\theta}}(\varnothing)\right]+j c_{1} v_{j}^{p}\right]-B_{j-1}(\varnothing) A_{j-1}^{1+\frac{1}{\theta}}(\varnothing)\left[A_{j-1}^{2}(\omega)[1-(\theta+\right.
\end{aligned}
$$




$$
\begin{aligned}
& \text { 1) } \left.\left.\left.B_{j-1}(\varnothing) A_{j-1}^{\frac{1}{\theta}}(\varnothing)\right]+(j-1) c_{1} v_{j}^{p}\right]\right]-\theta\left[A_{j-1}(\varnothing)-A_{j}(\varnothing)\right]^{-1}\left[B_{j}(\varnothing) A_{j}(\omega) A_{j}^{1+\frac{1}{\theta}}(\varnothing)-\right. \\
& \left.\left.\left.B_{j-1}(\varnothing) A_{j-1}(\omega) A_{j-1}^{1+\frac{1}{\theta}}(\varnothing)\right]^{2}\right\}\right\} \\
& I_{34}=-\frac{\partial^{2} \ln L(\underline{\Phi})}{\partial p \partial \theta}=\sum_{j=1}^{k} \ln v_{j}\left\{R _ { j } B _ { j } ( \varnothing ) A _ { j } ^ { \frac { 1 } { \theta } } ( \varnothing ) A _ { j } ( \omega ) \left[B_{j}(\varnothing) A_{j}^{\frac{1}{\theta}}(\varnothing)-\frac{1}{\theta} \ln A_{j}(\varnothing)-\ln (1+\right.\right. \\
& \left.\left.B_{j}(\varnothing)\right)\right]-\frac{\left(r_{j}+R_{j}\right) c_{2} v_{j}^{p}}{\theta^{2} A_{j}^{\frac{1}{\theta}}(\varepsilon)}\left[\ln A_{j}(\varepsilon)-\theta \ln \left(1+\frac{c_{2} v_{j}^{p}}{\theta}\right)+\frac{c_{2} v_{j}^{p}}{A_{j}^{\frac{1}{\theta}}(\varepsilon)}\right]-r_{j}\left[A_{j-1}(\varnothing)-\right. \\
& \left.A_{j}(\varnothing)\right]^{-1}\left\{B _ { j } ( \varnothing ) A _ { j } ( \omega ) A _ { j } ^ { 1 + \frac { 1 } { \theta } } ( \varnothing ) \left[(\theta+1)\left(B_{j}(\varnothing) A_{j}^{\frac{1}{\theta}}(\varnothing)-\ln \left(1+B_{j}(\varnothing)\right)\right)-\frac{1}{\theta} \ln A_{j}(\varnothing)-\right.\right. \\
& 1]-B_{j-1}(\varnothing) A_{j-1}(\omega) A_{j-1}^{1+\frac{1}{\theta}}(\varnothing)\left[(\theta+1)\left(B_{j-1}(\varnothing) A_{j-1}^{\frac{1}{\theta}}(\varnothing)-\ln \left(1+B_{j-1}(\varnothing)\right)\right)-\right. \\
& \left.\frac{1}{\theta} \ln A_{j-1}(\varnothing)-1\right]+\left[B_{j}(\varnothing) A_{j}^{1+\frac{1}{\theta}}(\varnothing) A_{j}(\omega)-B_{j-1}(\varnothing) A_{j-1}^{1+\frac{1}{\theta}}(\varnothing) A_{j-1}(\omega)\right][1- \\
& \theta\left[A_{j-1}(\varnothing)-A_{j}(\varnothing)\right]^{-1}\left(A_{j}(\varnothing)\left(\ln \left(1+B_{j}(\varnothing)\right)-B_{j}(\varnothing) A_{j}^{\frac{1}{\theta}}(\varnothing)\right)-A_{j-1}(\varnothing)(\ln (1+\right. \\
& \left.\left.\left.\left.\left.\left.B_{j-1}(\varnothing)\right)-B_{j-1}(\varnothing) A_{j-1}^{\frac{1}{\theta}}(\varnothing)\right)\right)\right]\right\}\right\}
\end{aligned}
$$




$$
\begin{aligned}
& I_{44}=-\frac{\partial^{2} \ln L(\underline{\Phi})}{\partial \theta^{2}} \\
& =\frac{1}{\theta} \sum_{j=1}^{k}\left\{R _ { j } B _ { j } ( \varnothing ) \left[A _ { j } ^ { \frac { 1 } { \theta } } ( \varnothing ) \left(1-B_{j}(\varnothing) A_{j}^{\frac{1}{\theta}}(\varnothing)+\frac{1}{\theta} \ln A_{j}(\varnothing)+\ln (1\right.\right.\right. \\
& \left.\left.\left.+B_{j}(\varnothing)\right)\right)-\left(1+B_{j}(\varnothing)\right)^{-1}\right] \\
& +\frac{\left(r_{j}+R_{j}\right) c_{2} v_{j}^{p}}{\theta^{2}}\left[A_{j}^{\frac{-1}{\theta}}(\varepsilon)\left(\frac{1}{\theta} \ln A_{j}(\omega)-\ln \left(1+\frac{c_{2} v_{j}^{p}}{\theta}\right)+\frac{c_{2} v_{j}^{p}}{\theta A_{j}^{\frac{1}{\theta}}(\varepsilon)}-1\right)\right. \\
& \left.+\left(1+\frac{c_{2} v_{j}^{p}}{\theta}\right)^{-1}\right] \\
& +r_{j}\left[A_{j-1}(\varnothing)-A_{j}(\varnothing)\right]^{-1}\left\{\left[A_{j}(\varnothing)\left(\ln \left(1+B_{j}(\varnothing)\right)-B_{j}(\varnothing) A_{j}^{\frac{1}{\theta}}(\varnothing)\right)^{2}\right.\right. \\
& +\frac{1}{\theta} B_{j}(\varnothing) A_{j}(\varnothing)\left[\left(1+B_{j}(\varnothing)\right)^{-1}\right. \\
& \left.\left.-A_{j}^{\frac{1}{\theta}}(\varnothing)\left[1-B_{j}(\varnothing) A_{j}^{\frac{1}{\theta}}(\varnothing)+\frac{1}{\theta} \ln A_{j}(\varnothing)+\ln \left(1+B_{j}(\varnothing)\right)\right]\right]\right] \\
& -A_{j-1}(\varnothing)\left(\ln \left(1+B_{j-1}(\varnothing)\right)-B_{j-1}(\varnothing) A_{j-1}^{\frac{1}{\theta}}(\varnothing)\right)^{2} \\
& +\frac{1}{\theta} B_{j-1}(\varnothing) A_{j-1}(\varnothing)\left[\left(1+B_{j-1}(\varnothing)\right)^{-1}\right. \\
& \left.-A_{j-1}^{\frac{1}{\theta}}(\varnothing)\left[1-B_{j-1}(\varnothing) A_{j}^{\frac{1}{\theta}}(\varnothing)+\frac{1}{\theta} \ln A_{j-1}(\varnothing)+\ln \left(1+B_{j-1}(\varnothing)\right)\right]\right] \\
& +\left[A_{j-1}(\varnothing)-A_{j}(\varnothing)\right]^{-1}\left(A_{j}(\varnothing)\left(\ln \left(1+B_{j}(\varnothing)\right)-B_{j}(\varnothing) A_{j}^{\frac{1}{\theta}}(\varnothing)\right)\right. \\
& \left.\left.\left.-A_{j-1}(\varnothing)\left(\ln \left(1+B_{j-1}(\varnothing)\right)-B_{j-1}(\varnothing) A_{j-1}^{\frac{1}{\theta}}(\varnothing)\right)\right)^{2}\right\}\right\}
\end{aligned}
$$

\section{EM- Algorithm}

EM-algorithm is broadly applicable approach to the iterative computation of maximum Likelihood estimates (MLE), it was described and analyzed by Dempster, Laird, and Rubin (1977). The EM methods can be explained most easily in terms of a random sample that consists of two components, one observed and one unobserved or missing. Missing data occurs in life-testing, when, for example, a number of electrical units are switched on and the time when each fails is recorded. In such an experiment, it is usually necessary to curtail the recordings prior to the failure of all units. The failure times of the 
units still working are unobserved, but the number of censored observations and the time of the censoring obviously provide information about the distribution of the failure times. Many common applications of EM methods do involve missing-data problems, but this is not necessary. Often, an EM method can be constructed based on two steps- called the Expectation step or the E-step and the maximization step or the M-step. Therefore, the algorithm is called the EM algorithm. The basic idea of the EM-algorithm is associate with the given incomplete-data, a complete-data problem. For which Maximum Likelihood (ML) estimation is computationally more tractable. For instance, the complete-data problem for chosen may yield a closed form solution to the (MLE) or may be amenable to MLE computation with a standard computer package. The methodology of the EM algorithm consists of reformulating the problem in terms of this more easily solved complete-data problem, establishing a relationship between the likelihoods of these two problems and exploiting the simpler MLE computation of the complete-data problem in the M-step of the iteration computing algorithm. Under k-level constant stress, the pdf of the truncated GL distribution will be applied in Q-function of EMalgorithm. Hence, For the E-step, we calculate $\Phi\left(\underline{\emptyset} \underline{\emptyset}_{m}\right)$, where $\underline{\emptyset}=\left(c_{1}, c_{2}, p, \theta\right)$, and $\widehat{\emptyset}_{m}=\left(\hat{c}_{1 m}, \hat{c}_{2 m}, \hat{p}_{m}, \hat{\theta}_{m}\right)$ in $m^{\text {th }}$ iteration. Thus, the Q-function of the truncated GL distribution can be obtained as follows,

$$
\Phi\left(\underline{\emptyset}, \underline{\emptyset}_{m}\right)=E\left(\ln L, \underline{\emptyset}_{m}\right)=\int \ln L f(t) d t,
$$

therefore, the Q-function is

$$
\begin{aligned}
& \Phi\left(\underline{\emptyset}, \underline{\emptyset}_{m}\right)=\sum_{j=1}^{k}\left\{R_{j} E\left(\ln A_{j}(\emptyset), \underline{\emptyset}_{m}\right)+\left(r_{j}+R_{j}\right) \ln A_{j}(\varepsilon)\right. \\
& \left.+r_{j} E\left(\ln \left[A_{j-1}(\varnothing)-A_{j}(\varnothing)\right], \underline{\emptyset}_{m}\right)\right\} \text {. }
\end{aligned}
$$

For the M-Step, we note that the maximization can be done by finding the solution of

$$
E\left(\frac{\partial \ln L}{\partial \emptyset}, \underline{\emptyset}_{m}\right)=\frac{\partial \Phi\left(\underline{\emptyset}, \underline{\emptyset}_{m}\right)}{\partial \emptyset}=0
$$

Then, we obtain the estimators of $\left(c_{1}, c_{2}, p, \theta\right)$ by differentiating Eq.(10) with respect to $\left(c_{1}, c_{2}, p, \theta\right)$ respectively and equating to zero. The $\mathrm{E}$ and $\mathrm{M}$ steps are repeatedly until the estimates of parameters converges. Therefore, we use the MAThCad program to solve the nonlinear equations simultaneously to obtain $\left(\hat{c}_{1}, \hat{c}_{2}, \hat{p}, \hat{\theta}\right)$ as will be seen in Section 5 .

\section{Numerical Results}

A numerical study is conducted to assess the performance of the proposed algorithm in Section (3) and Section (4). The a progressively Type-I censored grouped sample under constant stress test is considered to obtain the unknown parameters $\left(\hat{c}_{1}, \hat{c}_{2}, \hat{p}, \hat{\theta}\right)$ by solving the system of nonlinear equations get from Eqs. (6-9). Then the values which getten of estimators are used to get the absolute relative bias (ARBias), and mean square error (MSE). Furthermore, Fisher information matrix, the scale parameters and the reliability function. Tables (1) to (3) summarize the results of the simulation study based on different values of $N, R$ and $v_{1}=1, v_{2}=2$. 
From the results, the following observations can be made on the performance of CSALT parameter estimation of the two scales truncated GL lifetime distribution by EMalgorithm. We observe the maximum likelihood estimators of the parameters $c_{10}=$ $0.4, c_{20}=0.001, p_{0}=1.990, \theta_{0}=7.70$, have good statistical properties than the second set of parameters $c_{10}=0.4, c_{20}=0.001, p_{0}=1.993, \theta_{0}=7.51$ for the same sample size (see Table 1). Also, as the sample size increases the RABiases and MSEs of the estimated parameters decrease. This indicates that the maximum likelihood estimates provide asymptotically normally distributed and consistent estimators for the parameters. In addition, the asymptotic variances of the estimators are decreasing when the sample size increasing (see Table2). Moreover, the reliability function decreases when the mission time $x_{0}$ increases, and there is an inverse proportional relationship between $\hat{\alpha}_{1 u}$, $\hat{\alpha}_{2 u}$ and $\hat{R}_{u}\left(x_{0}\right)$ at the same mission time (see Table3).

Table 1: The MLE, ARBias, and MSE of the Parameters

\begin{tabular}{|c|c|c|c|c|c|c|c|c|c|c|}
\hline \multicolumn{6}{|c|}{$c_{10}=0.4, c_{20}=0.001, p_{0}=1.990, \theta_{0}=7.70$} & \multicolumn{5}{|c|}{$c_{10}=0.4, c_{20}=0.001, p_{0}=2.0, \theta_{0}=7.51$} \\
\hline para. & $\mathrm{N}$ & $R$ & $M L E$ & ARBias & $M S E$ & $\mathrm{~N}$ & $R$ & $M L E$ & ARBias & $M S E$ \\
\hline$c_{1}$ & 30 & 20 & 0.4005 & 0.0013 & 2.9E-7 & 66 & 42 & 0.4000 & 0.0009 & $1.3 \mathrm{E}-7$ \\
\hline$c_{2}$ & & & 0.0008 & 0.1559 & 2.4E-8 & & & 0.0009 & 0.0970 & 9.4E-9 \\
\hline $\mathrm{p}$ & & & 1.9793 & 0.0054 & $1.2 \mathrm{E}-4$ & & & 1.9880 & 0.0027 & $3.0 \mathrm{E}-5$ \\
\hline$\theta$ & & & 7.6509 & 0.0064 & $6.4 \mathrm{E}-3$ & & & 7.5320 & 0.0029 & 4.7E-4 \\
\hline \multicolumn{6}{|c|}{$c_{10}=0.4, c_{20}=0.001, p_{0}=1.993, \theta_{0}=7.51$} & \multicolumn{5}{|c|}{$c_{10}=0.4, c_{20}=0.001, p_{0}=1.993, \theta_{0}=7.51$} \\
\hline$M S E$ & $\mathrm{~N}$ & $R$ & $M L E$ & ARBias & $M S E$ & $\mathrm{~N}$ & $R$ & $M L E$ & ARBias & $M S E$ \\
\hline c1 & 30 & 20 & 0.40090 & 0.0022 & 8.0E-7 & 97 & 68 & 0.40000 & 3.7E-5 & $2.1 \mathrm{E}-10$ \\
\hline$c 2$ & & & 0.00083 & 0.1700 & 2.9E-8 & & & 0.00099 & 1.3 & $1.8 \mathrm{E}-12$ \\
\hline \multirow[t]{2}{*}{$\mathrm{p}$} & & & 1.98190 & 0.0056 & $1.2 \mathrm{E}-4$ & & & 2.00000 & 0.000063 & $1.6 \mathrm{E}-8$ \\
\hline & & & 7.59390 & 0.0112 & $7.0 \mathrm{E}-3$ & & & 7.51100 & 0.000100 & $6.2 \mathrm{E}-7$ \\
\hline \multicolumn{6}{|c|}{$c_{10}=0.4, c_{20}=0.001, p_{0}=2.0, \theta_{0}=6.54$} & \multicolumn{5}{|c|}{$c_{10}=0.4, c_{20}=0.001, p_{0}=1.992, \theta_{0}=7.52$} \\
\hline$M S E$ & $\mathrm{~N}$ & $R$ & $M L E$ & ARBias & $M S E$ & $\mathrm{~N}$ & $R$ & $M L E$ & ARBias & $M S E$ \\
\hline $\mathrm{c} 1$ & 47 & 30 & 0.40520 & 0.0130000 & $2.7 \mathrm{E}-5$ & 186 & 118 & 0.40080 & 0.0021 & $6.9 \mathrm{E}-7$ \\
\hline$c 2$ & & & 0.00099 & 0.0780000 & $6.1 \mathrm{E}-9$ & & & 0.00087 & 0.1313 & $1.7 \mathrm{E}-8$ \\
\hline \multirow[t]{2}{*}{$\mathrm{p}$} & & & 2.00000 & 0.0001929 & $1.5 \mathrm{E}-7$ & & & 1.99200 & 0.0036 & $5.1 \mathrm{E}-5$ \\
\hline & & & 6.51800 & 0.0034090 & $5.0 \mathrm{E}-4$ & & & 7.46850 & 0.0069 & $2.7 \mathrm{E}-3$ \\
\hline
\end{tabular}


Table 2: Estimated Variance-Covariance Matrix

\begin{tabular}{|c|c|c|c|c|c|c|c|c|c|c|c|}
\hline \multicolumn{2}{|c|}{$c_{10}=0.4$} & $c_{20}=0$ & $1, p_{0}=1$ & \multicolumn{2}{|c|}{$\theta_{0}=7.70$} & \multicolumn{6}{|c|}{$c_{10}=0.4, c_{20}=0.001, p_{0}=2.0, \theta_{0}=7.51$} \\
\hline$N$ & $R$ & $\hat{c}_{1}$ & $\hat{c}_{2}$ & $\hat{p}$ & $\hat{\theta}$ & $N$ & $R$ & $\hat{c}_{1}$ & $\hat{c}_{2}$ & $\hat{p}$ & $\hat{\theta}$ \\
\hline \multirow[t]{4}{*}{30} & 20 & 0.1441 & -0.9892 & 0.0184 & 0.00034 & 66 & 42 & 0.1971 & -0.7374 & 0.4070 & 0.0061 \\
\hline & & & 0.0791 & -0.1261 & -0.0237 & & & & 0.0739 & -0.1517 & -0.2770 \\
\hline & & & & $2.3 \mathrm{E}-3$ & 4.4E-5 & & & & & 0.0840 & 0.0013 \\
\hline & & & & & $8.2 \mathrm{E}-7$ & & & & & & $1.9 \mathrm{E}-5$ \\
\hline \multicolumn{6}{|c|}{$c_{10}=0.4, c_{20}=0.001, p_{0}=1.993$} & \multicolumn{6}{|c|}{$c_{10}=0.4, c_{20}=0.001, p_{0}=1.993, \theta_{0}=7.51$} \\
\hline$N$ & $R$ & $\hat{c}_{1}$ & $\hat{c}_{2}$ & $\hat{p}$ & $\hat{\theta}$ & $N$ & $R$ & $\hat{c}_{1}$ & $\hat{c}_{2}$ & $\hat{p}$ & $\hat{\theta}$ \\
\hline \multirow[t]{4}{*}{30} & 20 & 0.138 & -0.9881 & 0.0170 & $0.4 \mathrm{E}-4$ & 97 & 68 & 0.1192 & -0.1286 & 0.526 & 0.0093 \\
\hline & & & 0.0776 & -0.1218 & -0.0248 & & & & 0.0940 & -0.161 & -0.0029 \\
\hline & & & & 0.0021 & $-4.3 \mathrm{E}-5$ & & & & & 0.066 & 0.0011 \\
\hline & & & & & $8.7 \mathrm{E}-7$ & & & & & & $2.1 \mathrm{E}-5$ \\
\hline \multicolumn{6}{|c|}{$c_{10}=0.4, c_{20}=0.001, p_{0}=2.0$, } & \multicolumn{6}{|c|}{$c_{10}=0.4, c_{20}=0.001, p_{0}=1.992, \theta_{0}=7.52$} \\
\hline$N$ & $R$ & $\hat{c}_{1}$ & $\hat{c}_{2}$ & $\hat{p}$ & $\hat{\theta}$ & $N$ & $R$ & $\hat{c}_{1}$ & $\hat{c}_{2}$ & $\hat{p}$ & $\hat{\theta}$ \\
\hline \multirow[t]{4}{*}{47} & 30 & 0.1224 & -0.2969 & 0.147 & 0.0034 & 186 & 118 & 0.1292 & -0.3192 & 0.8824 & 0.0175 \\
\hline & & & 0.0200 & -0.357 & 0.0083 & & & & 0.0397 & -0.3862 & -0.7677 \\
\hline & & & & 0.0180 & 0.0004 & & & & & 0.1068 & 0.0021 \\
\hline & & & & & $0.1 \mathrm{E}-6$ & & & & & & $4.2 \mathrm{E}-5$ \\
\hline
\end{tabular}

Table 3: Estimated $\widehat{\alpha}_{1 u}, \quad \widehat{\alpha}_{2 u}$ and $\widehat{R}_{u}\left(x_{0}\right)$

\begin{tabular}{|c|c|c|c|c|c|c|c|c|c|c|c|}
\hline \multicolumn{6}{|c|}{$c_{10}=0.4, c_{20}=0.001, p_{0}=1.990, \theta_{0}=7.70$} & \multicolumn{6}{|c|}{$c_{10}=0.4, c_{20}=0.001, p_{0}=2.0, \theta_{0}=7.51$} \\
\hline$N$ & $R$ & $\hat{\alpha}_{1 u}$ & $\hat{\alpha}_{2 u}$ & $x_{0}$ & $\hat{R}_{u}\left(x_{0}\right)$ & $N$ & $R$ & $\hat{\alpha}_{1 u}$ & $\hat{\alpha}_{2 u}$ & $x_{0}$ & $\hat{R}_{u}\left(x_{0}\right)$ \\
\hline \multirow[t]{4}{*}{30} & 20 & 0.2267 & 0.00048 & 10 & 0.9949 & 66 & 42 & 0.226 & 0.00051 & 10 & 0.9946 \\
\hline & & & & 25 & 0.8572 & & & & & 25 & 0.8501 \\
\hline & & & & 30 & 0.6249 & & & & & 30 & 0.6108 \\
\hline & & & & 40 & 0.0240 & & & & & 40 & 0.0213 \\
\hline \multicolumn{6}{|c|}{$c_{10}=0.4, c_{20}=0.001, p_{0}=1.993, \theta_{0}=7.51$} & \multicolumn{6}{|c|}{$c_{10}=0.4, c_{20}=0.001, p_{0}=1.993, \theta_{0}=7.51$} \\
\hline$N$ & $R$ & $\hat{\alpha}_{1 u}$ & $\hat{\alpha}_{2 u}$ & $x_{0}$ & $\hat{R}_{u}\left(x_{0}\right)$ & $N$ & $R$ & $\hat{\alpha}_{1 u}$ & $\hat{\alpha}_{2 u}$ & $x_{0}$ & $\hat{R}_{u}\left(x_{0}\right)$ \\
\hline \multirow[t]{4}{*}{30} & 20 & 0.2267 & 0.00047 & 10 & 0.9950 & 97 & 68 & 0.225 & 0.00056 & 10 & 0.9940 \\
\hline & & & & 25 & 0.8593 & & & & & 25 & 0.8400 \\
\hline & & & & 30 & 0.6295 & & & & & 30 & 0.5900 \\
\hline & & & & 40 & 0.0253 & & & & & 40 & 0.0180 \\
\hline \multicolumn{6}{|c|}{$c_{10}=0.4, c_{20}=0.001, p_{0}=2.0, \theta_{0}=6.54$} & \multicolumn{6}{|c|}{$c_{10}=0.4, c_{20}=0.001, p_{0}=1.992, \theta_{0}=7.52$} \\
\hline$N$ & $R$ & $\hat{\alpha}_{1 u}$ & $\hat{\alpha}_{2 u}$ & $x_{0}$ & $\hat{R}_{u}\left(x_{0}\right)$ & $N$ & $R$ & $\hat{\alpha}_{1 u}$ & $\hat{\alpha}_{2 u}$ & $x_{0}$ & $\hat{R}_{u}\left(x_{0}\right)$ \\
\hline \multirow[t]{4}{*}{47} & 30 & 0.2280 & 0.00052 & 10 & 0.9943 & 186 & 118 & 0.226 & 0.00049 & 10 & 0.9948 \\
\hline & & & & 25 & 0.8384 & & & & & 25 & 0.8556 \\
\hline & & & & 30 & 0.5839 & & & & & 30 & 0.6225 \\
\hline & & & & 40 & 0.0166 & & & & & 40 & 0.0242 \\
\hline
\end{tabular}




\section{Conclusion}

Truncated distributions arise when sample selection is not possible in some sub-region of the sample space. For example, the GLD is considered inappropriate for modeling lifetime data because left hand side of its distribution extends to negative infinity, and this could conceivably result in modeling negative times-to-failure. This has necessitated the use of truncated GLD truncated at point zero for modeling lifetime data. So, this paper is assumed that the stress is effected of two scale parameters of the truncated generalized logistic distribution, and dealt with constant-stress accelerated life testing in the case of progressive Type-I censored grouped data. Maximum likelihood estimators of the model parameters and the associated Fisher Information matrix are derived by used the EM algorithm method. Also, the estimator of the two scale parameters, and the reliability function under usual conditions are derived.

\section{References}

1. Aggarwala, R. (2001). Progressive Interval Censoring: Some Mathematical Results with Applications to Inference. \{Communications in Statistics Theory and Methods 1935-1921,(9-8)30.

2. Attia, A. F., Aly, H. M., and Bleed, S. O. (2011a). Estimating and Planning Accelerated Life Test using Constant Stress for Generalized Logistic Distribution under Type-I Censoring. \{ISRN Applied Mathematics\}, vol. 2011, Article ID 203618, 15 pages.

3. Attia, A. F., Aly, H. M., and Bleed, S. O. (2011b). Estimating and Planning Accelerated Life Test using Constant Stress for Generalized Logistic Distribution under Type-II Censoring. \{Accepted at the 46th\} Annual Conference on Statistics, Computer Sciences and Operations Research, ISSR, Cairo University.

4. Balakrishnan, N., and Aggarwalla, R. (2000). Progressive Censoring Theory, Methods, and Application: Statistics for Industry and Technology\}. Birkhauser, Boston, Mass, U.S.A.

5. Dempster, A. P., Laird, N. M., and Rubin, D.B. (1977). Maximum Likelihood from Incomplete Data via the EM Algorithm. Journal of Royal Statistics Society Serial B Methodology, 39: 1-38.

6. Ding, C., Yang, C., and Siu-Keung, T. (2010). Accelerated Life Test Sampling Plans for the Weibull Distribution under Type-I Progressive Interval Censoring with Random Removals. \{Journal of Statistical Computation and Simulation\}, 80(8), 903-914.

7. Johnson, N. L., Kotz, S., and Balakrishnan, N. (1995). Continuous Univariate Distributions. John Wiley and Sons, New York.

8. Mann, N. R., Schafer, R. E., and Singpurwalla, N. D. (1974). Methods for Statistical Analysis of Reliability and Life Data. Wiley, New York.

9. Meeker, W. Q., and Nelson, W. B. (1975). Optimum Censored Accelerated Life Tests for Weibull, Extreme Value Distributions. IEEE Transaction on Reliability, 24, 321-332. 
10. Meeker, W. Q., and Hahn, G. J. (1977). Asymptotically Optimum Over-Stress Tests to Estimate the Survival Probability at a Condition with a Low Expected Failure Probability. Technometrics,.399-381

11. Molenberghs, G., and Verbeke, G. (2011). On The Weibull-Gamma Frailty Model, Its Infinite Moments, and Its Connection to Generalized Log-Logistic, Logistic, Cauchy, and Extreme Value Distributions. \{Journal of Statistical Planning and Inference, 14 861-868.

12. Nadarajah, S. and Kotz, S. (2005). A generalized Logistic Distribution. International Journal of Mathematics and Mathematical Sciences, 19, 3169-3174.

13. Nelson, W. B. (1990). Accelerated Life Testing: Statistical Models, Test Plan and Data Analysis. John Wiley and Sons, New York.

14. Tang, L. C., Sun, Y. S., Goh, T. N., and Ong, H. L. (2002). Planning MultipleLevels Constant-Stress Accelerated Life Tests. \{Annual Reliability and Maintainability Symposium \}, 338-342.

15. Tang, L. C., and Xu, K. (2005). A Multiple Objective Framework for Planning Accelerated Life Tests. \{IEEE Transactions on Reliability\}, 54(1), 58-63.

16. Yang, G. B. (1994). Optimum Constant Stress Accelerated Life Test Plans. \{IEEE Transactions on Reliability $\},\{43\}, 575-581$.

17. Yang, G. B., and Jin, L. (1994). Best Compromise Test Plans for Weibull Distribution with Different Censoring Times. Qual. Reliab. Eng. Int., 10, 411415. 